\title{
Restoration of endodontically treated teeth
}

\author{
F. Mannocci*1 and J. Cowie ${ }^{1}$
}

IN BRIEF
- Stresses that the endodontically treated
tooth needs to be restored back to form,
function and aesthetics.
Highlights the choices available to
restore both anterior and posterior teeth.
Provides an update on contemporary
adhesive techniques

The restoration of endodontically treated teeth has undergone significant changes in the last 20 years. Most of these
changes are associated with the preservation of tooth structure, this has been achieved first of all with the increasing use
of operative microscopes, nickel titanium instruments and more recently cone beam computed tomography; these instru-
ments have allowed the clinicians to reduce significantly the amount of coronal and radicular hard tooth tissue removed
in the process of cutting access cavities. The use of composites has also allowed the clinicians to restore with adhesive
techniques teeth that would otherwise require extensive and destructive mechanical retentions. The use of partial crowns
is becoming increasingly popular and this also helps prevent tooth structure loss. This article will focus on the choices
available to restore both anterior and posterior teeth and will focus more on these contemporary adhesive techniques.

The completion of root canal treatment does not signal the end of patient management. The endodontically treated tooth needs to be restored back to form, function and aesthetics. The quality of the coronal restoration will directly impact on the survival and success of the endodontically treated tooth. The provision of a restoration with a good coronal seal has been suggested to reduce the risk of failure of a root canal treated tooth by reducing bacterial microleakage into the recently cleaned, shaped, and filled root canal system. ${ }^{1}$

Additionally, provision of a well-executed restoration will return the tooth to form and function, re-establish proximal contacts and occlusal stability as well as protecting the tooth from future breakdown, both noncarious (fracture) and carious.

The restoration of endodontically treated teeth has changed in recent years. The availability of adhesive techniques has increased the clinician's repertoire in terms of restoring teeth. Amalgam cores and cast metal posts are being replaced by direct composite and fibre-posts, all ceramic crowns and composite resin crowns are often chosen because of their superior aesthetic outcome.

This article will focus on the choices available to restore both anterior and posterior

'King's College Dental Institute, London, SE1 9RT ${ }^{*}$ Correspondence to: Dr Francesco Mannocci

Email: francesco.mannocci@kcl.ac.uk

\section{Refereed Paper}

Accepted 28 January 2014

DOI: 10.1038/sj.bdj.2014.198

${ }^{\circ}$ British Dental Journal 2014; 216: 341-346 teeth and will focus more on these contemporary adhesive techniques.

\section{EFFECTS OF ENDODONTIC TREATMENT ON THE TOOTH}

A tooth that requires endodontic treatment is commonly a tooth that has lost a large volume of tooth tissue and is heavily restored. These teeth are often more prone to fracture. The fracture of endodontically treated teeth may range from a simple cusp fracture all the way to catastrophic root fracture requiring extraction. The loss of marginal ridge/s has been shown to reduce cuspal stiffness. In the case of the MOD cavity, this was to an extent of $63 \%{ }^{2}$ In a more recent study using microcomputed tomography a significant reduction in tooth stiffness was noted with an access cavity preparation and more so with a post preparation for a cast metal post. The preparation for a fibre-post proved more conservative and less tooth tissue needed to be removed. ${ }^{3}$

The medicaments and irrigants used during root canal treatment can alter the physical properties of dentine and the prolonged use of calcium hydroxide renders the dentine more brittle and prone to fracture. ${ }^{4,5}$ Additionally, non-vital teeth lose proprioception and are less adept at perceiving increased load. ${ }^{6}$

Preservation of coronal tooth tissue without compromising endodontic access is desirable. Adhesive techniques allow the clinician to add to existing, residual tooth tissue and do not require creation of macromechanical retention; this permits preservation rather than removal of hard tooth structure.

\section{TIMING OF THE RESTORATIVE PROCEDURE}

The factors to consider in terms of timing of the restorative phase of treatment are:

- Pre-existing endodontic status

- Quality of root canal filling

- Position of tooth in the mouth

- Type of restoration planned.

If root canal treatment has been completed to a technically satisfactory standard and the tooth is symptoms free then it is sensible to proceed with the final restoration straight away. This is particularly true when dealing with a previously vital, uninfected tooth. If the tooth was symptomatic that is, tender to biting and on lateral pressure, then delaying the final restoration for a few weeks while the tooth settles would be prudent. If the tooth fails to settle then root canal retreatment may well be required.

If the tooth had a small pre-existing periapical radiolucency (less than $2 \mathrm{~mm}$ ) then the tooth should be treated in the same manner as the vital tooth. If the tooth had a larger pre-operative periapical radiolucency and a good root filling has been completed then a short review period should be considered.

There is conflicting evidence whether the pre-operative lesion size has an effect on endodontic success; some authors report it makes no difference, ${ }^{7,8}$ while others suggest a larger lesion has a negative effect on outcome. ${ }^{9,10}$ A larger lesion might indicate the presence of a higher bacterial load within the root canal. In this scenario a more conservative review approach should be taken 
to ascertain whether the root canal treatment has been successful. A review period is sensible especially in a practice setting where patients will be financially investing in the final indirect restoration and are usually less accepting of failure.

In those teeth where the prognosis is doubtful and a good root filling has been executed it may be advisable to allow for a longer review period until there is clinical evidence, and in some case, radiographic evidence, of healing. Should the clinician take this approach then the tooth must be adequately protected during this period to prevent unwanted, catastrophic tooth fracture. It is sensible to place a plastic restoration with cuspal protection or stabilise the tooth with a well-fitting, well-burnished thin copper band or an orthodontic band cemented with a glass-ionomer cement.

\section{SURVIVAL OF THE ENDODONTICALLY TREATED TOOTH}

Endodontically treated teeth have a good survival rate. Indeed an epidemiological study with a sample size of $1,462,936$ teeth recalled at eight years showed a 97\% survival rate. Interestingly, of the teeth that were lost $85 \%$ had had no full coronal coverage restoration. ${ }^{11}$ This was corroborated by a more recent study, which showed a four-year survival rate of 95\% and again teeth with cast restorations survived better than those without. ${ }^{9}$ This study also indicated that a terminal tooth in the arch has a lower survival rate and teeth with proximal contacts both mesially and distally have a higher survival rate. They also showed that presence of a cast post and core may be associated with a decreased survival of an endodontically treated tooth.

The majority of root filled teeth (61.4\%) that fail, fail owing to restorative reasons for example, non-restorable caries rather than endodontic failure per se. ${ }^{12}$ In fact, only some $12 \%$ of cases failed because of endodontic failure. Additionally, $8.8 \%$ of teeth failed due to vertical root fracture. The importance of attention to detail with the coronal restoration and cuspal protection shouldn't be underestimated. It has been reported that a molar tooth has a six-fold increase risk of failure when left without a cuspal coverage cast restoration. ${ }^{13}$ In a more recent systematic review, endodontically treated teeth restored with crowns have a higher longterm survival rate ( $81 \pm 12 \%$ after ten years) compared with teeth without crown coverage (63 $\pm 15 \%$ after ten years). ${ }^{14}$ This review also showed that in the first three years the survival rate of root filled teeth without crowns was satisfactory $(84 \% \pm 9 \%)$ but this significantly dropped off after this period. This falls into line with two randomised clinical trials on endodontically treated premolars restored with crown coverage..$^{15,16}$ Teeth that were restored with fibre-posts and composite were more effective than amalgam in preventing root fracture but less effective in preventing secondary caries. ${ }^{15}$ In summary, the overwhelming body of evidence suggests cast restorations especially on posterior teeth increase tooth survival after endodontic treatment.

\section{RESTORATION TYPE}

The type of restoration chosen for a root filled tooth will depend on the remaining hard tooth structure available. The amount of tooth remaining will dictate the fracture resilience of a tooth and how the restoration will need to be retained. It follows that the preservation of as much tooth tissue as possible will improve likely outcome. There are different challenges in restoring the anterior and posterior dentition. The posterior dentition undergoes much higher forces when eating and chewing and is more susceptible to fracture. Anterior teeth are less prone to fracture but from a patient perspective the aesthetic demand is greater.

\section{Anterior teeth}

\section{Composite resin restoration}

In anterior teeth that are minimally to moderately restored then a direct composite restoration will be the restoration of choice. The composite may be placed directly over the gutta percha, which should ideally be cut back to osseous level, some clinicians prefer to use a glass-ionomer base or dualcure composite base where it can be difficult to light-cure composite. Placing composite below the level of the cemento-enamel junction not only provides a good coronal seal but can reduce the fracture susceptibility of the tooth (Fig 1). ${ }^{17}$

This is a very useful technique for teeth that have suffered trauma in a young patient where the root canal walls are thin. Figure 2 demonstrates obturation of a very wide apex case with Biodentine and subsequent restoration of a tooth with a fibre-post and composite build-up using a palatal silicone matrix made from a pre-operative diagnostic wax-up in a 12-year-old patient. Direct composite even for larger restorations of anterior teeth is often the restoration of choice in the developing dentition as it can be easily maintained, offers good aesthetics and enables crown restorations to be deferred until the gingival shape is stable.

Composite achieves a good seal owing to its ability to bond to tooth structure; it has good physical properties and can be selected
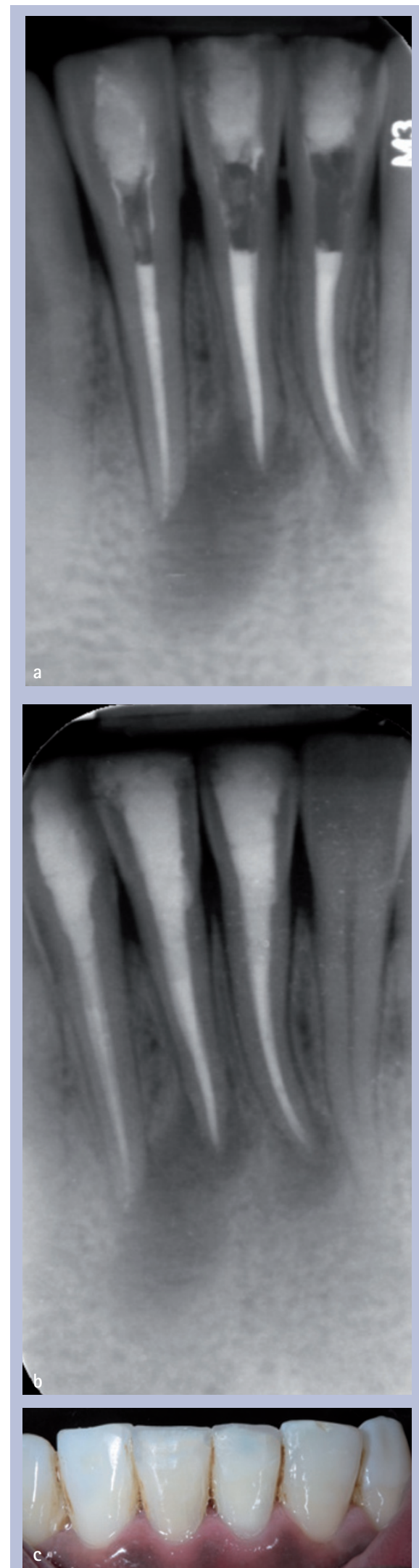

Fig. 1 Restoration of lower right lateral, central and lower left central incisors after root canal retreatment. (a) Post-obturation radiograph showing gutta percha finished at osseous level; (b) Post-restoration radiograph after complete restoration with composite including incisal edge build-ups; (c) Post-restoration photograph showing incisal tip build-ups 


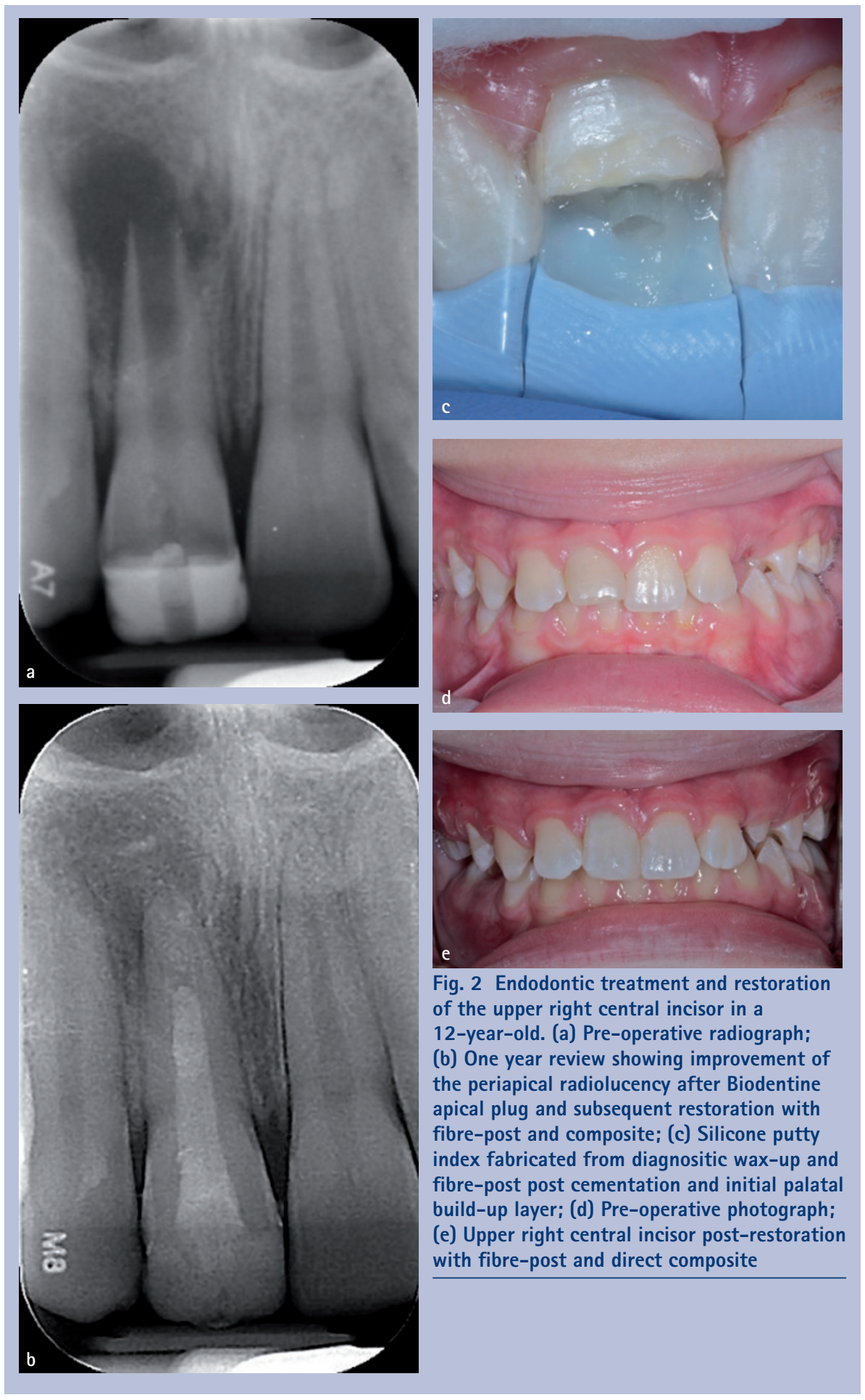

by shade and polished to achieve a good aesthetic result. It is possible to internally bleach discoloured teeth before composite placement to achieve excellent aesthetic results.

\section{Ceramic or composite resin veneers}

Veneers normally cover the entire labial surface of the tooth including the incisal edge and through to the proximal contacts. Ceramic or composite resin veneers are seldom recommended for endodontically treated anterior teeth as it is not easy to incorporate the access cavity within such restorations and often the tooth tissue loss surface area to bond to.

\section{Metal-ceramic crowns}

Metal-ceramic crowns are commonly prescribed when an anterior, endodontically treated tooth is to be crowned and represent the main non-adhesive restoration of the anterior dentition. A reduction of the labial surface of approximately 1.8-2 mm is necessary. This reduction may compromise the strength of the remaining tooth tissue; so caution should be exercised before prescribing such a restoration. Far from preserving residual tooth structure, it may actually promote its loss. It is quite sensible to consider full metal coverage palatally to minimise the reduction on that aspect but in an increasingly cosmetic era some patients are less inclined to proceed with this option. In general, crowning of anterior teeth is indicated if the amount of tooth structure left is not sufficient for a direct restoration and for aesthetic reasons.

\section{All-ceramic crowns}

All-ceramic crowns offer the clinician a superior aesthetic result with often a reduced tooth preparation when compared to a metal-ceramic crown. Some all-ceramic crowns can allow for a labial preparation of 1-1.5 mm, for example IPS eMax crowns. Tooth preparation must be very precise with good rounded internal line angles so as not to concentrate stress under the crown, which can lead to micro-crack formation and fracture propagation. These crowns can be adhesively cemented.

\section{Posterior teeth}

\section{Amalgam restoration}

Amalgam has been used as a restorative material with good long-term success. In recent years it has lost popularity among some clinicians and particularly with patients over concerns regarding the toxic effect of metal ions released by the amalgam overtime, but primarily owing to its cosmetic shortcomings. However, amalgam functions very well as a restorative material as it has a high compressive strength and contrary to some, it has a safe, successful clinical history. ${ }^{17}$

Amalgam is non-adhesive and when used conventionally without cuspal coverage leads to a higher risk of cusp or root fracture. ${ }^{18}$ For this reason cuspal coverage is advocated. In a study on the long-term survival of extensive amalgam restorations that involved the rebuilding of cusps the cumulative survival rate was $88 \%$ at 100 months. ${ }^{19}$

Molar teeth rarely require a post unless means a significant reduction in available there has been significant loss of tooth structure. A coronal-radicular core build-up with silver amalgam utilising the pulp chamber, and possible $2 \mathrm{~mm}$ canal extensions, has proved very effective in vitro and in vivo. ${ }^{20}$ This is the classically termed Nayyar core technique. ${ }^{21}$

When employing this technique and indeed when using adhesive composite resin direct cores the placement of dentine pins is not advocated. Pins can cause areas of micro-stress and micro-cracks during placement, which may propagate and weaken the tooth. ${ }^{22}$ 


\section{Composite resin restoration}

Composite resin restorations are rarely acceptable as definitive long-term restorations for posterior teeth. Invariably, posterior teeth undergoing endodontic treatment have lost significant amounts of tooth structure. Extensive loss approximally and the deep access cavity can make it difficult to restore the tooth to good anatomical shape and function. This is often complicated by the need to overlay cusps to reduce the chance of short- to mid-term cusp/tooth fracture. It may be acceptable to accept composite as a definitive restoration where the access cavity is limited to just the occlusal surface. Most commonly, composite resin is used to buildup a core filling before subsequent crowning of the tooth. This can be with an incrementally placed, light-cure composite or with a dual-cure composite resin. The light-cure version is technically very challenging especially when small increments are being placed to extend into the root canal space, bulk placement is not advocated as lightcuring deep into the tooth is unpredictable. The dual-cure option is more straightforward but requires the use of fine tips to inject the composite to the gutta percha interface to prevent unwanted voids within the composite. Composite resin cores placed in this Nayar fashion are termed composite dowelcores. If auxiliary retention is required then a fibre-post can be placed and composite built-up immediately.

\section{Gold onlays and crowns}

Gold restorations have stood the test of time and are renowned for their durability. ${ }^{23}$ The gold onlay enables preservation of sound tooth structure as the preparation is conservative, this may infer greater strength for the endodontically treated tooth. Gold is still the material of choice for posterior teeth but this tends to be where aesthetics are not a major concern. Upper second molars are good candidates for these types of restoration or restorations where interocclusal space is limited or patients are bruxists. Gold onlay preparation should include cuspal coverage of all cusps.

\section{Composite resin and ceramic onlays/crowns}

The onlay preparation differs little from that used for vital teeth. The internal line angles should be rounded, the preparation walls slightly flare, rather than a chamfer preparation there is normally a $90^{\circ}$ shoulder finish. A minimum preparation depth of $1.5 \mathrm{~mm}$ and the proximal boxes should extend above the contact point. Cuspal coverage is again advocated to reduce the risk of tooth fracture. Often gingivectomy with electrosurgery

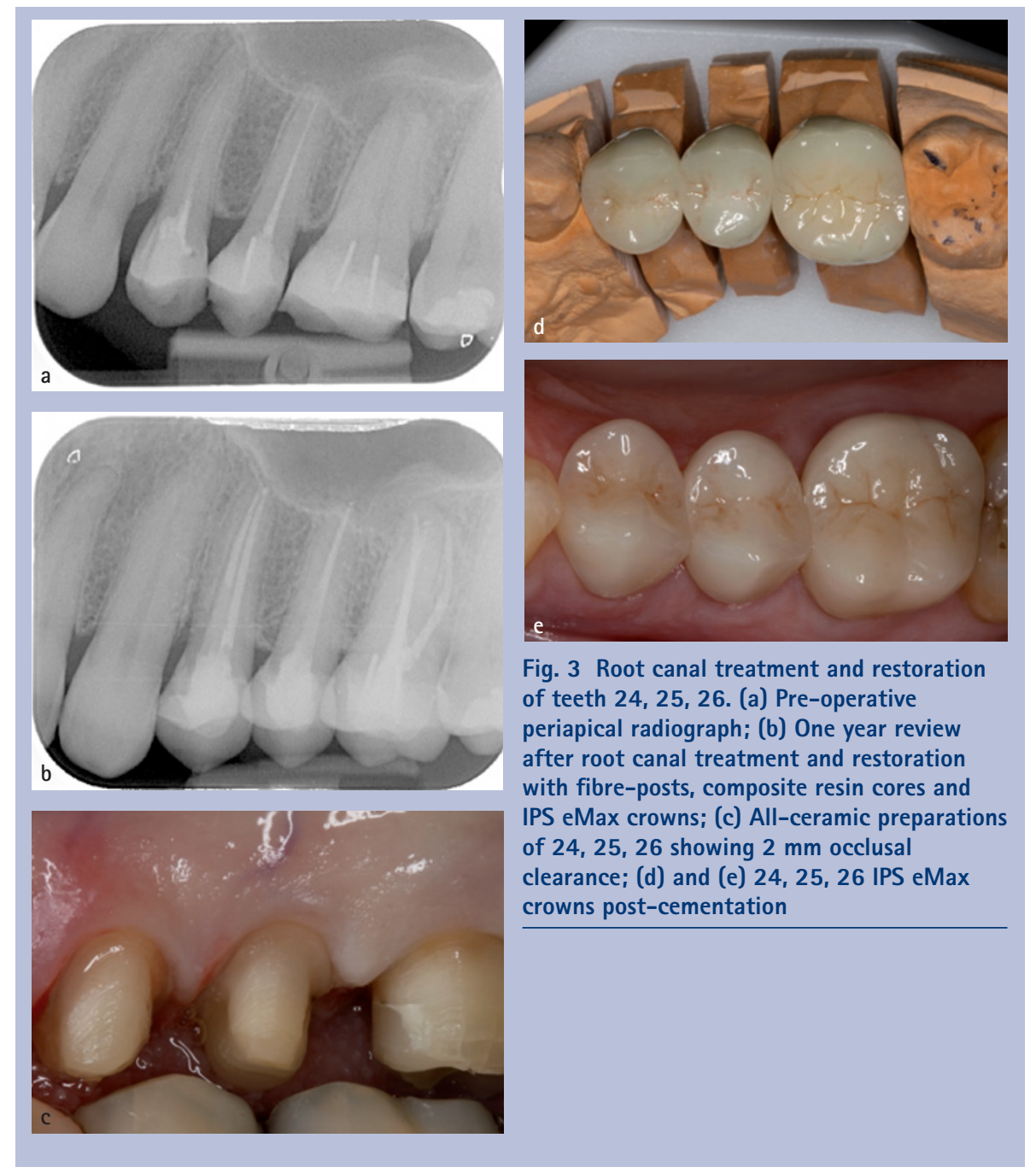

in the interproximal region can help clarify finishing margins and impression taking. A composite or glass-ionomer restoration should be placed directly over the gutta percha to seal the root filling and to help creation of the correct preparation form. This permits the preparation design to have a flat base and to block out any undercuts that would impede impression taking and accurate seating of the final restoration. Ceramic onlay/crowns are normally cemented with adhesive resins.

All-ceramic crowns are not really suitable in posterior teeth because of the risk of fracture; although they are sometimes used in premolars for aesthetic reasons. Despite the robust nature of zirconia as a coping for crowns the plane of weakness of these crowns is at the interface between this coping and the laminated porcelain. There are those that advocate the use of ceramic in its monolithic state and suggest this reduces the risk of this type of fracture. ${ }^{24}$ Again, as eluded to earlier in this article the nature of the modern patient may well press the clinician to use metal-free restorations and all-ceramic crowns are an alternative to metal-ceramic crowns, Figure 3 shows the use of these restorations in the posterior region.

There is no clear evidence to favour but composite resin onlays/ crowns are, in general, less expensive and easier to repair.

\section{Metal-ceramic crowns}

Metal-ceramic crowns are the most commonly placed full coverage restoration in the posterior dentition. Metal-ceramic crowns may also be used as bridge abutments. Unfortunately the conventional approach to preparing metal-ceramic crowns requires an extensive heavy tooth reduction to create sufficient room for the restoration. To overcome some of these issues an adjustment to this preparation could be considered. The non-aesthetic elements of the crown can be finished in metal with metal occlusal coverage, this enables a more conservative preparation in these areas and as a consequence preservation of valuable tooth structure. The full metal-collar finish is an underused finishing margin for these types of crown and can be invaluable for teeth with difficult restorability where a true shouldertype finish would be most difficult, this is ceramic or composite resin onlays/crowns, 


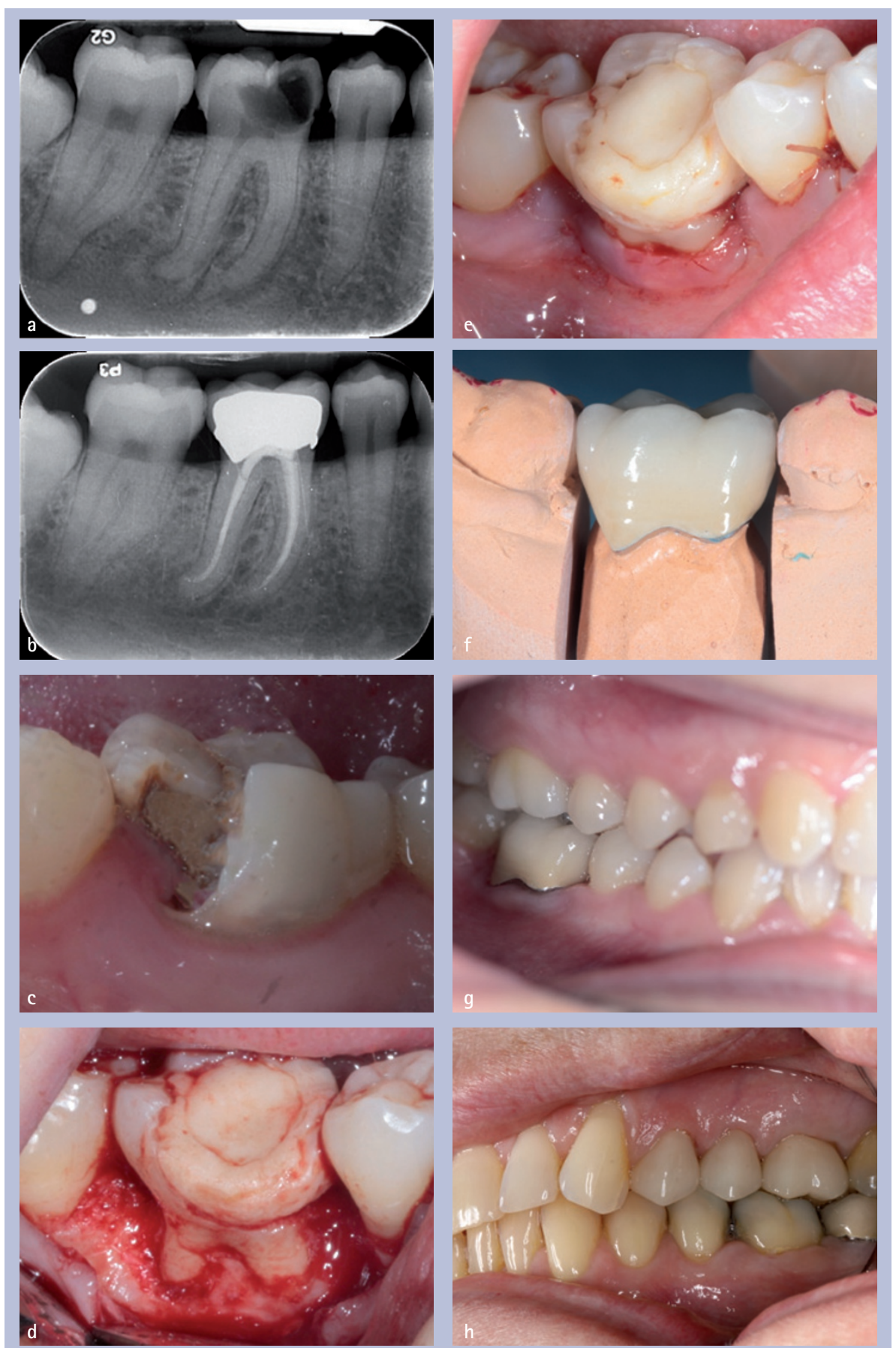

Fig. 4 Root canal treatment, crown lengthening and restoration of tooth 46 with a metalceramic crown. (a) Pre-operative radiograph 46; (b) One year review radiograph after root canal treatment and restoration of tooth 46; (c) Pre-operative view demonstrating deep subgingival caries; (d) Crown lengthening surgery with gingivectomy; (e) Immediately postsurgery; ( $f$ ) 46 crown preparation showing circumferential chamfer finish for metal collar; (g) and (h) 46 metal-ceramic crown with metal collar

particularly true when preparing in the delicate furcation area. Figure 5 demonstrates the use of this restoration in combination with an adhesive composite dowel-core as previously described.

\section{POSTS}

\section{Indications for posts}

In the restoration of endodontically treated teeth the placement of a post is generally reported the increased probability of survival. In other words, there were more teeth not restored with a fibre post lost due to crown or root fractures.

A recent literature review on clinical studies of fibre posts reported that fibre-reinforced composite posts out-perform metal posts in the restoration of endodontically treated teeth; however, the evidence cannot be considered as conclusive. ${ }^{27}$ The placement of a fibre-reinforced composite post would seem to protect against failure, especially under conditions of extensive coronal destruction; the most common type of failure with fibre-reinforced composite posts is debonding. In terms of post diameter, ordinarily the preparation of the root canal will have created a sufficient diameter to place a post of adequate diameter. This concept is particularly true of fibre posts where the post is chosen to fit the root canal rather than the tooth prepared to fit a post, which is the case for many indirect, cast-post systems. It is also worth noting that the remaining root canal filling should not fall below $3 \mathrm{~mm}$. The relative frequency of periapical lesions increases significantly when this is the case. ${ }^{28}$

The available evidences do not rule out the use of cast posts; however, since the use of cast posts may result in a significantly greater loss of tooth structure compared to fibre posts, ${ }^{3}$ their use should be limited to those cases in which no additional dentine has to be removed to allow for their cementation. Many cases may need endodontic retreatments, in this respect fibre posts are often difficult to remove, however, their removal is achieved by trophing through the post without any additional removal of root or crown dentine.

\section{Fibre posts}

Studies have shown that the mechanical properties of carbon, glass and quartz fibre posts are substantially similar; for this reason, the more aesthetic glass and quartz fibre posts have now replaced carbon fibre posts. The modulus of elasticity of fibre posts is generally lower than that of metal posts. ${ }^{29}$ The main difference, in terms of mechanical properties between fibre and metal posts is the loss of flexural strength that affects fibre posts that are exposed to cyclic loading in a wet environment or thermocycled..$^{30}$ As a result of this, the mode of failure of fibre post restored teeth is unlikely to be root fracture but normally decementation that may or may not be associated with the development of caries at the interface between the tooth and the restoration. The adhesion of the fibre posts to the composite core is mainly micromechanical. The 
irregularities on the surface of the post provide the retention for the bonding resin.

There are many techniques available to bond and cement fibre posts. As with all adhesive dentistry this should be carried out with the use of rubber dam. Once the gutta percha has been removed, care should be taken to clean the root canal space free of residual gutta percha and sealer using an ultrasonic or piezon scaler. A fibre post should be selected and cut to length so that it will be contained in its entirety within the composite restoration. One of the more widely accepted techniques involves etching the root canal with 37\% phosphoric acid followed by washing and drying the canal with sterile paper points and cementation with a dual cure resin cement applied with a fine tip to allow placement deep in to the root canal. The post is then placed into the canal and light-cured. A composite core can then be placed as needed and in a conventional manner.

\section{CONCLUSION}

In summary, the preservation of tooth structure is critical to the survival of an endodontically treated tooth. Adhesive techniques allow the clinician to preserve rather than remove dentine and in conjunction with exacting attention to detail can provide the basis of long-lasting, aesthetic restorations.

1. Saunders W P, Saunders E M. Coronal leakage as a cause of failure in root-canal therapy: A review. Dent Traumatol 1994; 10: 105-108.

2. Reeh E S, Messer H H, Douglas W H. Reduction in tooth stiffness as a result of endodontic and restorative procedures. J Endod 1989; 15: 512-516.

3. Ikram O H, Patel S, Sauro S, Mannocci F.
Micro-computed tomography of tooth tissue volume changes following endodontic procedures and post space preparation. Int Endod J 2009; 42: 1071-1076.

4. Andreasen J O, Farik B, Munksgaard E C. Long-term calcium hydroxide as a root canal dressing may increase risk of root fracture. Dent Traumatol 2002; 18: 134-137.

5. Grigoratos D, Knowles J, Ng Y L, Gulabivala K. Effect of exposing dentine to sodium hypochlorite and calcium hydroxide on its flexural strength and elastic modulus. Int Endod J 2001: 34: 113-119.

6. Randow K, Glantz P O. On cantilever loading of vita and non-vital teeth. An experimental clinical study. Acta Odontol Scand 1986; 44: 271-277.

7. Bystrom A, Happonen R P, Sjogren U, Sundqvist $\mathrm{G}$. Healing of periapical lesions of pulpless teeth after endodontic treatment with controlled asepsis. Endod Dent Traumatol 1987: 3: 58-63.

8. Sjogren U, Hagglund B, Sundqvist G, Wing K. Factors affecting the long-term results of endodontic treatment. J Endod 1990; 16: 498-504.

9. $\mathrm{Ng}$ Y L, Mann V, Gulabivala K. A prospective study of the factors affecting outcomes of non-surgical root canal treatment: Part 2: Tooth survival. Int Endod J 2011; 44: 610-625.

10. Hoskinson S E, Ng Y L, Hoskinson A E, Moles D R, Gulabivala K. A retrospective comparison of outcome of root canal treatment using two different protocols. Oral Surg Oral Med Oral Pathol Oral Radiol Endod 2002; 93: 705-715.

11. Salehrabi R, Rotstein I. Endodontic treatment outcomes in a large patient population in the USA: An epidemiological study. J Endod 2004; 30: 846-850.

12. Zadik Y, Sandler V, Bechor R, Salehrabi R. Analysis of factors related to extraction of endodontically treated teeth. Oral Surg Oral Med Oral Pathol Oral Radiol Endod 2008; 106: e31-35.

13. Aquilino S A, Caplan D J. Relationship between crown placement and the survival of endodontically treated teeth. J Prosthet Dent 2002; 87: 256-263.

14. Stavropoulou A, Koidis P. A systematic review of single crowns on endodontically treated teeth. J Dent 2007: 35: 761-767.

15. Mannocci F, Bertelli E, Sherriff M, Watson T F, Ford T R. Three-year clinical comparison of survival of endodontically treated teeth restored with either full cast coverage or with direct composite restoration. J Prosthet Dent 2002; 88: 297-301.

16. Mannocci F, Qualtrough A J, Worthington H V, Watson T F, Pitt Ford T R. Randomized clinical comparison of endodontically treated teeth restored with amalgam or with fibre posts and resin composite: Five-year results. Oper Dent 2005; 30: 9-15.

17. Dental amalgam: Update on safety concerns. ADA council on scientific affairs. J Am Dent Assoc 1998; 129: 494-503

18. Hansen E K, Asmussen E, Christiansen NC. In vivo fractures of endodontically treated posterior teeth restored with amalgam. Endod Dent Traumatol 1990; 6: 49-55.

19. Plasmans P J, Creugers N H, Mulder J. Long-term survival of extensive amalgam restorations. J Dent Res 1998; 77: 453-460.

20. Plasmans P J, Visseren L G, Vrijhoef M M, Kayser A F. In vitro comparison of dowel and core techniques for endodontically treated molars. J Endod 1986; 12: 382-387.

21. Nayyar A, Walton R E, Leonard L A. An amalgam coronal-radicular dowel and core technique for endodontically treated posterior teeth. J Prosthet Dent 1980; 43: 511-515.

22. Ersöz E. Evaluation of stresses caused by dentin pin with finite elements stress analysis method. J oral Rehabil 2000; 27: 769-773.

23. Smales R J, Hawthorne W S. Long-term survival of extensive amalgams and posterior crowns. J Dent 1997; 25: 225-227.

24. Guess P C, Zavanelli R A, Silva N R, Bonfante E A, Coelho P G, Thompson V P. Monolithic CAD/CAM lithium disilicate versus veneered Y-TZP crowns: Comparison of failure modes and reliability after fatigue. Int J Prosthodont 2010; 23: 434-442.

25. Ferrari M, Cagidiaco M C, Grandini S, De Sanctis $M$, Goracci C. Post placement affects survival of endodontically treated premolars. J Dent Res 2007; 86: 729-734.

26. Cagidiaco M C, Garcia-Godoy F, Vichi A, Grandini S, Goracci C, Ferrari M. Placement of fibre prefabricated or custom made posts affects the 3-year survival of endodontically treated premolars. Am J Dent 2008; 21: 179-184.

27. Cagidiaco M C, Goracci C, Garcia-Godoy F, Ferrari M. Clinical studies of fibre posts: A literature review. Int J Prosthodont 2008; 21: 328-336.

28. Kvist $T$, Rydin $E_{\text {, Reit }} \mathrm{C}$. The relative frequency of periapical lesions in teeth with root canal-retained posts. J Endod 1989; 15: 578-580.

29. Plotino G, Grande N M, Bedini R, Pameijer C H, Somma F. Flexural properties of endodontic posts and human root dentin. Dent Mater 2007; 23: 1129-1135.

30. Mannocci F, Sherriff M, Watson T F. Three-point bending test of fibre posts. J Endod 2001; 27: 758-761. 